\title{
FREE LIE SUBALGEBRAS OF THE COHOMOLOGY OF LOCAL RINGS
}

BY

\author{
LUCHEZAR L. AVRAMOV
}

\begin{abstract}
A criterion is established, in terms of the Massey products structure carried by the homology of partial resolutions, for the Yoneda cohomology algebra $\operatorname{Ext}_{A}(k, k)$ to be a free module over the universal envelope of a free graded Lie subalgebra. It is shown that several conjectures on the (co)homology of local rings, in particular on the asymptotic behaviour of the Betti numbers, follow from such a structure. For all rings with $\operatorname{edim} A-\operatorname{depth} A \leqslant 3$, and for Gorenstein rings with $\operatorname{edim} A-\operatorname{depth} A=4$, the following dichotomy is proved: Either $A$ is a complete intersection, or $\operatorname{Ext}_{A}(k, k)$ contains a nonabelian free graded Lie subalgebra.
\end{abstract}

0. Ten conjectures on the homology of local rings (in place of Introduction). In this paper $(A, \mathfrak{m}, k)$ will denote a noetherian local ring $A$ with maximal ideal $\mathfrak{m}$ and residue field $k$. A well-known homological invariant of $A$ is its Poincaré series

$$
P_{A}(t)=\sum_{i=0}^{\infty} b_{i} t^{i} \in \mathbf{Z} \llbracket t \rrbracket,
$$

where $b_{i}\left(=b_{i}(A)\right)$ is the $i$ th Betti number of $A$, i.e. the rank of the $i$ th free module in the unique, up to noncanonical isomorphism, $A$-free resolution $X$ of $k$. Much of the work on the homology of local rings has been motivated by the following famous conjecture:

$\mathrm{C}_{0} \cdot P_{A}(t)$ represents a rational function.

Recently this has been settled in the negative by D. Anick, ${ }^{1}$ who constructed for each $k$ a $k$-algebra $A$ requiring 5 generators for $\mathfrak{m}$ and having $\mathfrak{m}^{3}=0$, with transcendental Poincare series. On the other hand, more than 20 years of research have produced different kinds of conditions, which ensure the rationality of $P_{A}(t)$. Before collecting for further reference several of them in a theorem, we introduce some notation, to be kept for the rest of the paper.

$\operatorname{edim} A=$ embedding dimension of $A:=\operatorname{dim}_{k} \mathfrak{m} / \mathfrak{m}^{2}\left(=b_{1}(A)\right)$;

$\operatorname{dim} A=$ Krull dimension of $A$;

depth $A=$ maximal length of an $A$-regular sequence in m;

$d(A)=$ complete intersection defect of $A:=\operatorname{dim}_{k} a / \mathfrak{n a}-(\operatorname{dim} R-\operatorname{dim} A)$, where $(R, \mathfrak{n})$ is a regular local ring such that $R / \mathfrak{a}$ is isomorphic to the m-adic

Received by the editors November 11, 1980.

1980 Mathematics Subject Classification. Primary 13H10, 13D25; Secondary 13D99, 55T20.

Key words and phrases. Yoneda products, Massey products, homology of local rings, deviations, local complete intersections.

' D. Anick, Counter-examples to a conjecture of Serre, Thesis, Massachusetts Institute of Technology, June 1980; cf. also D. Anick, C. R. Acad. Sci. Paris Ser. A-B 290 (1980), A729-A732. 
completion of $A$; note that $d(A) \geqslant 0$, the rings for which equality holds being called complete intersections. ${ }^{2}$

THEOREM 0.1. Under each one of the following conditions $P_{A}(t)$ represents a rational function:

(1) $A$ is a complete intersection (Tate, Zariski, 1957);

(2) $\operatorname{edim} A-\operatorname{dim} A \leqslant 1$ [Sh];

(3) $\operatorname{edim} A-\operatorname{depth} A \leqslant 2$ [Sc];

(4) $\operatorname{edim} A-\operatorname{depth} A=3$ and $A$ is Gorenstein [W];

(5) $\operatorname{edim} A-\operatorname{depth} A=3$ and $d(A)=1$ [Go 2; Av 2];

(6) edim $A=3$ and $\mathrm{m}^{3}=0$ (Backelin and Fröberg, 1978);

(7) $\operatorname{dim}_{k} \mathrm{~m}^{2} / \mathfrak{m}^{3} \leqslant 1$ (J. Sally, 1978; Backelin, 1978).

The striking disparity of these conditions leaves little hope for a satisfactory ring-theoretic characterization of the local rings with rational Poincare series. The purpose of this section is to review several conjectures, formulated in terms of Betti numbers, deviations, and Yoneda products structure in cohomology, which can be organized around three main themes:

(i) the already acquired understanding that the complete intersections are remarkably disjoint from the rest of the local rings by their (co)homological behaviour (cf. $\mathrm{C}_{1}, \mathrm{C}_{3}, \mathrm{C}_{4}(\mathrm{~b})$, and $\mathrm{C}_{9}$ below);

(ii) the asymptotic properties of the Betti numbers $\left(\mathrm{cf} . \mathrm{C}_{1}, \mathrm{C}_{2}, \mathrm{C}_{10}\right)$;

(iii) the fact that the homological invariants, and especially the deviations, give a certain hold on the singularity of $A$, and there are situations when this "should not get worse": this is the content of $\mathrm{C}_{4}-\mathrm{C}_{8}$.

It should be emphasized that for some of these statements the status of open questions would have been more appropriate. However, for the sake of more precise formulations and unity of exposition, we have chosen, taking the usual risks, to have conjectures throughout.

Let $r_{A}$ be the radius of convergence of $P_{A}(t)$. It is infinite if and only if $A$ is regular, otherwise one has the double inequality $0<r_{A} \leqslant 1$. The upper bound holds for all infinite series in $\mathbf{Z} \llbracket t \rrbracket$, while the lower one is a consequence of the coefficientwise inequality of power series (denoted $\ll$ ), due to Serre:

$$
P_{A}(t) \ll \frac{(1+t)^{\operatorname{edim} A}}{1-\sum_{i=1}^{\operatorname{edim} A-\operatorname{depth} A} c_{i} t^{i+1}}
$$

where $c_{i}=\operatorname{dim}_{k} H_{i}(K)$ (cf. §3A below).

$\mathrm{C}_{1}$ (Golod, Gulliksen). If $r_{A} \geqslant 1$, then $A$ is a complete intersection. (The converse is true by 0.5 below.)

From the product formula for the Poincare series (cf. (0.4)) it follows that for $n>\operatorname{edim} A$ the function $n \mapsto b_{n}(A)$ is nondecreasing (if one restricts to nonregular rings, then this is true for $n \geqslant 0$ ). The set of all almost everywhere nondecreasing

\footnotetext{
${ }^{2}$ L. L. Avramov, Math. Ann. 228 (1977), 27-37.
} 
functions from the set $\mathbf{N}$ of nonnegative integers to the nonnegative reals $\mathbf{R}^{+}$has a partial preorder: $f \leqslant g$ if and only if there exist positive integers $p$ and $q$ such that for all $n \in \mathbf{N}, f(n) \leqslant p g(q n)$. The equivalence class of $f$ for the relation $f \sim g \Leftrightarrow(f$ $\preccurlyeq g$ and $g \preccurlyeq f$ ) is called the growth of $f$ and denoted [ $f$ ] (for details cf. [BK]). We write $\leqslant$ for the induced partial order on the factor set.

EXAMPLE. For any real numbers $a>1, b>1, c>d>0$, one has the inequalities $\left[a^{n}\right]=\left[b^{n}\right] \geqslant\left[n^{c}\right] \geqslant\left[n^{d}\right]$. The growth of $f$ is said to be polynomial (resp. exponential) if there exists a $c \in \mathbf{N}$ for which $[f]=\left[n^{c}\right]\left(\operatorname{resp}\right.$. if $\left.[f]=\left[2^{n}\right]\right)$. Note $[f]=\left[2^{n}\right]$ implies the existence of $b>1, m \in \mathbf{N}$, such that $f(n) \geqslant b^{n}$ for all $n \geqslant m$.

Define $b_{A}: \mathbf{N} \rightarrow \mathbf{R}^{+}$by setting $b_{A}(n)=b_{n}(A)$. From $(0.2)$ and $(0.4)$ one has the double inequality

$$
\left[n^{\operatorname{edim} A-\operatorname{dim} A-1}\right] \leqslant\left[b_{A}\right] \leqslant\left[2^{n}\right]
$$

(in the regular case, i.e. when $\operatorname{edim} A=\operatorname{dim} A$, the left-hand term is defined to be $[0]$ ). By a theorem of Gulliksen [Gu], complete intersections are characterized by the fact that the growth of $b_{A}$ is at most polynomial.

$\mathrm{C}_{2}[\mathrm{Av} 4]$. Either $A$ is a complete intersection, or $b_{A}$ has exponential growth.

In the study of the Betti numbers, the main tools are the very rich algebraic structures carried by $X \otimes k=\operatorname{Tor}^{A}(k, k)=T^{A}$, and by its graded $k$-space dual $\operatorname{Hom}_{A}(X, k)=\operatorname{Ext}_{A}(k, k)=E_{A}$. In the sequel we shall asume $E_{A}$ is equipped with the product opposite (in the graded sense) to Yoneda's. It becomes a Hopf algebra when given the diagonal obtained by dualizing Cartan and Eilenberg's product $m$ in $T^{A}$ (for details cf. [GL]). We denote by $I(\quad)$ the kernel of the natural augmentation of $\left(\right.$ ) onto $k$, and by ()$_{+}$the subset of homogeneous elements of positive even degree. The algebra $T^{A}$ has divided powers $\gamma_{n}$, compatible with its diagonal (cf. [GL]). Denote by $L_{A}$ the subspace of $E_{A}$, orthogonal to $T_{0}^{A} \oplus I^{(2)}\left(T^{A}\right)$, where $I^{(2)}\left(T^{A}\right)=I^{2}\left(T^{A}\right)+\sum_{n \geqslant 2} k \cdot \gamma_{n}\left(T^{A}\right)_{+}$, and set $Q\left(T^{A}\right)=I\left(T^{A}\right) / I^{(2)}\left(T^{A}\right)$. Assuming that in characteristic 2 all Lie algebras are "adjusted" in the sense of Sjödin [Sj], we have the following fundamental structure theorem due to Milnor and Moore, André, and Sjödin.

THEOREM 0.3 [An], [Sj]. The subspace $L_{A}$ is a graded Lie subalgebra of $E_{A}$ (endowed with the commutator structure), and, as a Hopf algebra, $E_{A}$ is isomorphic to the universal enveloping algebra $U\left(L_{A}\right)$.

Note that a local homomorphism $(A, \mathfrak{m}, k) \rightarrow(B, \mathfrak{n}, l)$ induces a homomorphism of graded Lie algebras $L_{B} \rightarrow L_{A} \otimes l$. Also, $L_{A}$ is closely connected to the cohomology of the cotangent complex of the $A$-algebra $k$. There is a canonical homomorphism of graded Lie algebras $H^{*}(A, k, k) \rightarrow L_{A}$, which, by results of Quillen and André is an isomorphism in degrees $\leqslant \pi(A)$, where $\pi(A)=2 p$ when char $k=p>0$, and $\pi(A)=\infty$ when char $k=0$. A consequence of the Poincaré-Birkhoff-Witt theorem for $L_{A}$ is the equality of formal power series

$$
P_{A}(t)=\prod_{j=0}^{\infty} \frac{\left(1+t^{2 j+1}\right)^{e_{2 j+1}}}{\left(1-t^{2 j+2}\right)^{e_{2 j+2}}}
$$


which uniquely determines the integers $e_{i}=\operatorname{dim}_{k} L_{A}^{i} ; e_{i}$ is classically called the $(i-1)$ th deviation of $A$ and denoted $\varepsilon_{i-1}$.

The next theorem illustrates the ubiquitous appearance of the complete intersections in the theory.

THEOREM 0.5. Each one of the following conditions is necessary and sufficient for $A$ to be a complete intersection:

(1) $P_{A}(t)=(1+t)^{n} /\left(1-t^{2}\right)^{r}$; in this case $n=\operatorname{edim} A$, and $n-r=\operatorname{dim} A$ (Tate, Zariski, 1957);

(2) $e_{3}=0$ (Assmus, 1959);

(3) $e_{4}=0$ [GL];

(4) $e_{i}=0$ for $i \geqslant 3$;

(5) $e_{i}=0$ for all sufficiently large $i$ (Gulliksen, 1971);

(6) $e_{i}=0$ for all sufficiently large even $i[\mathbf{G u}$;

(7) $b_{n}(A) \leqslant f(n)$ for some $f \in \mathbf{Z}[t][\mathbf{G u}$;

(8) $L_{A}$ is nilpotent and finitely generated (Sjödin, 1976).

The rigidity of the deviations is an open question of long standing (cf. [GL, Remark on p. 154]).

$\mathrm{C}_{3}$. If $e_{i}=0$ for some $i \geqslant 1$, then $A$ is a complete intersection.

A new type of deviation has recently been introduced by André: being a series in $1+t \mathbf{Z} \llbracket t \rrbracket$, the Poincare series of $A$ can be written uniquely in the form

$$
P(t)=\frac{(1+t)^{e_{1}}}{\prod_{i \geqslant 2}\left(1-t^{i}\right)^{f_{i}}} .
$$

Note that $f_{2}=e_{2}, f_{i}=e_{i}-e_{i / 2}$ for $i \equiv 2(\bmod 4)$ and $i>2, f_{i}=e_{i}$ for $i \not 2$ $(\bmod 4)$. Considering the case of residual characteristic 2 , André ${ }^{3}$ has shown that $f_{i} \geqslant 0$ for all $i$. Gulliksen [ $\mathbf{G u}$ ] has formulated as an open question in all characteristics, and verified in several cases when an explicit formula for $P_{A}(t)$ is available, a strengthened version of both André's result and $\mathrm{C}_{3}$.

$\mathrm{C}_{3}^{\prime}$. If $A$ is not a complete intersection, then $f_{i}(A)>0$ for all $i \geqslant 2$.

The next group of conjectures, mostly based on work of Gulliksen, André, and the author, reflects the fact that the deviations measure the singularity of $A$; cf. Theorem 0.5 and the characterization of regular local rings by the vanishing of $e_{2}$.

Set

$$
\chi_{r}(A)=\sum_{j=1}^{r}(-1)^{j-1} e_{j}(A) .
$$

$\mathrm{C}_{4}$. (a) $\operatorname{dim} A-\chi_{2 i}(A) \geqslant 0$ for all $i \geqslant 0$;

(b) if equality holds in (a), then $A$ is a complete intersection (the converse is true by Theorem 0.5 );

(c) for any prime $\mathfrak{p} \subset A$, there is inequality: $\operatorname{dim} A_{\mathfrak{p}}-\chi_{2 i}\left(A_{\mathfrak{p}}\right) \leqslant \operatorname{dim} A-$ $\chi_{2 i}(A)$.

\footnotetext{
${ }^{3}$ M. André, Manuscripta Math. 18 (1976), 83-108.
} 
$\mathrm{C}_{5}$. For any prime $\mathfrak{p} \subset A$ and for all $r>0$, there is inequality:

$$
(-1)^{r-1} \chi_{r}(A) \geqslant(-1)^{r-1}\left(\chi_{r}\left(A_{\mathfrak{p}}\right)+\operatorname{dim} A / \mathfrak{p}\right) .
$$

$\mathrm{C}_{6}$. For any prime $\mathfrak{p} \subset A, e_{i}\left(A_{p}\right) \leqslant e_{i}(A)$ for $i \geqslant 1$.

$\mathrm{C}_{7}$. Let $A \rightarrow B$ be a flat local homomorphism. Then $e_{i}(A) \leqslant e_{i}(B)$ for $i \geqslant 1$.

$\mathrm{C}_{8}$. Let $(A, \mathfrak{m}, k) \rightarrow(B, \mathfrak{n}, l)$ be a flat local homomorphism. Then the induced map of Lie algebras $L_{B} \rightarrow L_{A} \otimes_{k} l$ is surjective in degrees $>1$.

Proposition 0.6. The following implications hold:

$$
\begin{aligned}
& \mathrm{C}_{4}(\mathrm{c}) \Leftarrow \mathrm{C}_{5} \Rightarrow \mathrm{C}_{6} \Leftarrow \mathrm{C}_{7} \Leftarrow \mathrm{C}_{8} ; \\
& \left.\begin{array}{c}
\mathrm{C}_{4}(\mathrm{a}) \text { for artinian rings and } \\
\mathrm{C}_{4}(\mathrm{c}) \text { for complete rings }
\end{array}\right\} \Rightarrow \mathrm{C}_{4}(\mathrm{a}) \text { and } \mathrm{C}_{4}(\mathrm{c}) \text {. }
\end{aligned}
$$

The proof is given in $\S 5$.

We describe briefly the present status of these conjectures. In a classical paper [L], Lech established $\mathrm{C}_{7}$ for $i=1$, and also proved $\mathrm{C}_{5}$ for all $A$ and $r=1$. Under the additional assumption that (i) $A$ is a homomorphic image of a regular local ring, Gulliksen [Gu] proved $\mathrm{C}_{5}$ for $r<\pi(A)$. The map of Lie algebras of $\mathrm{C}_{8}$ has been shown by the author ${ }^{4}$ to be surjective in all even degrees, and this has been extended by André $^{5}$ to all degrees $\neq 1$, assuming char $k=2$. Much evidence is available for $\mathrm{C}_{6}$. It is proved for all $A$ if either $i=1[\mathbf{L}]$ or $i$ is even ${ }^{6}$; furthermore, it is known to hold for all $i$ under any one of the following conditions: (i) as above; (ii) $A$ is universally japanese; (iii) $\mathfrak{p}$ is analytically unramified; (iv) $2 \in \mathfrak{p}$ (the first three cases are treated by the author ${ }^{7}$, and the last one by André ${ }^{8}$ ). Conjecture $\mathrm{C}_{4}$ is known for $i=1$ : noting that $\operatorname{dim} A-\chi_{2}(A)=d(A)$, (a) becomes Krull's altitude theorem, (b) is the Cohen-Macaulay theorem, and (c) is a conjecture of Kiehl and Kunz, proved by the author'.

We conclude with two statements on the Yoneda product structure in cohomology, which, in view of Theorem 0.3 , is the finest homological invariant of $A$.

$\mathrm{C}_{9}$ (Roos). If $\operatorname{Ext}_{A}(k, k)$ is a noetherian $k$-algebra, then $A$ is a complete intersection. (The converse is true by Theorem 0.5 .)

$\mathrm{C}_{10}$. If $A$ is not a complete intersection, then $L_{A}$ contains a nonabelian free graded Lie subalgebra $L$.

PROPOSITION 0.7. The following implications hold:

$$
\mathrm{C}_{0} \Rightarrow \mathrm{C}_{1} \Leftarrow \mathrm{C}_{2} \Leftarrow \mathrm{C}_{10} \Rightarrow \mathrm{C}_{9} \text {. }
$$

The proof is deferred to $\S 5$.

\footnotetext{
${ }^{4}$ See footnote 2.

${ }^{5} \mathrm{M}$. André, Gulliksen-Avramov exact sequences in characteristic two, Preprint, EFP Lausanne, 1979.

${ }^{6}$ See footnote 2.

${ }^{7}$ See footnote 2 .

${ }^{8}$ See footnote 5 .

${ }^{9}$ See footnote 2 .
} 
1. The main results. Our purpose in this paper is to establish a criterion, in terms of the Massey products structure of partial resolutions, for the validity of $\mathrm{C}_{10}$, and to verify its conditions for a class of rings, for which the rationality of the Poincare series is not known (compare Theorem 0.1). These results have been announced without proofs in $[\mathbf{A v} 4]$.

Theorem 1.1. Suppose $A$ satisfies the inequality $\operatorname{edim} A-\operatorname{depth} A \leqslant 4$, with equality admitted only for Gorenstein rings. Then either $A$ is a complete intersection, or $L_{A}$ contains a nonabelian free graded Lie subalgebra. In the latter case, $\left[b_{A}\right]=\left[2^{n}\right]$.

The proof is carried out in $\S 3$. After some further elaborations, worked out in $\S 4$, we obtain, for the class of rings considered here, strengthened versions of $C_{1}$ and $C_{2}$, and also partial results on the validity of $\mathrm{C}_{3}$.

THEOREM 1.2. Under the hypotheses of the previous theorem, the following conditions are equivalent:

(i) $A$ is a complete intersection;

(ii) the growth of $b_{A}$ is not exponential;

(iii) $r_{A}>\sqrt{2} / 2$;

(iv) $e_{6 i}(A)=0$ for some $i>0$;

(v) $e_{2 i}(A)=0$ for some $i>0$, and $A$ is Cohen-Macaulay;

(vi) $E_{A}$ is a noetherian $k$-algebra.

For the rest of this paper we denote by $X$ a fixed minimal $A$-free resolution of $k$, equipped with its standard structure of DG $\Gamma$-algebra (i.e algebra with a system of divided powers). For details we refer to [GL]. Consider a DG subalgebra $F \subset X$ satisfying the following conditions:

(i) $F_{i}=X_{i}$ for $i=0,1$ (i.e. $F$ contains the Koszul complex $K$ of $A$ );

(ii) $F$ is closed under the divided powers of $X$;

(iii) $X^{\#}$ is free as a $F^{\sharp}$-module, where ()$^{\#}$ denotes the forgetful functor assigning to a DG object its underlying graded object.

The set of all elements of $I H(F)$, decomposable in terms of matric Massey products, is denoted $M H(F)$. It is proved in [M] that this is a vector subspace (even an ideal) in $I H(F)$; we refer to May's paper for the definitions and basic properties of matric Massey products. The following convenient formalism has been introduced by Gulliksen.

Definition. The possibly infinite set $S=\left\{f_{i} \in I H(F) \mid i \in I\right\}$ is said to admit a trivial Massey operation (t.M.o.), if there exists a function $\gamma$, defined on the set of finite sequences of elements of $S$ (with repetitions) and taking values in $I F$, subject to the following conditions:

(i) $\gamma\left(f_{i}\right)=z_{i}$, with $\left[z_{i}\right]=f_{i}$ for each $i \in I$;

(ii)

$$
d \gamma\left(f_{i_{1}}, \ldots, f_{i_{n}}\right)=\sum_{j=1}^{n-1} \bar{\gamma}\left(f_{i_{1}}, \ldots, f_{i_{j}}\right) \cdot \gamma\left(f_{i_{j+1}}, \ldots, f_{i_{n}}\right) .
$$


(Here and in what follows, homology classes, cycles, etc. are always supposed homogeneous; square brackets denote the homology class of a cycle; if $a \in F_{m}$, then $\bar{a}=(-1)^{m+1} a$.)

THEOREM 1.4. Let $S=\left\{f_{i}\right\}_{i \in I}$ be a set of homology classes of $I H(F)$, such that:

(1) $S$ admits a trivial Massey operation; and

(2) the images of $\left\{f_{i}\right\}_{i \in I}$ in $I H(F) / M H(F)$ are linearly independent.

Then $L_{A}$ contains a free graded Lie subalgebra on a set of generators $\left\{e_{i} \in\right.$ $\left.L_{A}^{\operatorname{deg}\left(f_{i}\right)+1}\right\}_{i \in I}$.

The proof of this result occupies $\S 2$.

Corollary 1.5. Suppose $A=B /\left(m_{1}, \ldots, m_{s}\right)$, where $B$ is an arbitrary local ring and the $m_{i}$ 's are monomials in the elements of a $B$-regular sequence $x_{1}, \ldots, x_{r}$. If grade $\left(m_{1}, \ldots, m_{s}\right) \neq s$, then $L_{A}$ contains a free Lie subalgebra on two generators of degree 2 .

Note that the rationality of $P_{A}(t)$ is not known even for the local rings with “monomial relations", i.e. for $A$ 's obtained as above from a regular $B$. The next corollary is a sharpening of the structure theorem for the cohomology of Golod rings proved in [Av 1, (5.1)] and is extendable to the case of Golod homomorphisms. Recall that $A$ is said to be Golod if the right-hand side inequality in (0.2) becomes an equality or, equivalently, if some (resp. any) $k$-basis of $I H(K)$ admits a t.M.o. [Go 1].

Corollary 1.6. The ring $A$ is Golod if and only if the Lie subalgebra of $L_{A}$ generated by its elements of degree $\geqslant 2$ is isomorphic to the free Lie algebra on $\operatorname{sIH}(K)^{*}$ ( $s$ denotes the degree shifting functor $\left.(s V)_{i}=V_{i-1}\right)$.

(1.7) Remarks on Theorem 1.4. (1) Conditions (1) and (2) should be viewed as the proper homological substitutes for the algebraic notions of zero-divisors and indecomposable elements respectively. In this connection, note that if the ring $A$ admits as a subset of a minimal set of generators for $m$ elements $t_{1}, \ldots, t_{m}$ such that $t_{i} t_{j}=0$ for $1 \leqslant i, j \leqslant m$, then it is well known that $L_{A}$ contains a free Lie subalgebra on $m$ generators of degree 1 . In fact, a slight modification of our proof can produce a variant of the theorem, including this result as a degenerate case, when $F=A$. However, the interest of our theorem lies in the fact that even for integral domains $A$, zero-divisors abound in the homology of the strictly skew-commutative algebra $F$.

(2) The first condition is satisfied in each of the following cases: (a) the cycles $z_{i}$ representing $f_{i}$ can be chosen in such a way that $z_{i} z_{j}=0$ for $i, j \in I$; (b) $f_{i} f_{j}=0$ for $i, j \in I$, and $H_{q}(F)=0$ for $q>3 \min \left\{\operatorname{deg} f_{i} \mid i \in I\right\}$.

(3) If $H_{q}(F)=0$ for $0<q<m$, then $M H(F)=I^{2} H(F)$ in dimensions $\leqslant 3 m$.

(4) Our result can be viewed as an algebraic counterpart to a theorem of Chen [Ch], which gives conditions for the Pontriagin ring of a loop space to contain a free subalgebra. 


\section{Proof of Theorem 1.4.}

A. Free $k$-models.

Definition 2.1. A DG $k$-algebra $V$ is called a $k$-model of $F$ if there exists a finite sequence of skew-commutative DG algebras $F=U_{1}, U_{2}, \ldots, U_{t}=V$ and a sequence of homomorphisms of DG rings $u_{1}, u_{2}, \ldots, u_{t-1}$, where $u_{j}$ maps $U_{j}$ to $U_{j+1}$ or $U_{j+1}$ to $U_{j}$, such that $H\left(u_{j}\right)$ are isomorphisms for $j=1, \ldots, t-1$. The $k$-model is said to be free if, as an algebra, $V$ is isomorphic to a tensor product of symmetric algebras on even-dimensional generators and exterior algebras on odd-dimensional generators.

Note that each $k$-model defines an isomorphism $\alpha: H(F) \rightarrow H(V)$. The existence of free $k$-models will be deduced from the following very general statement.

LeMMA 2.2. Let $u_{0}: R \rightarrow A$ be a surjective homomorphism of commutative rings and let $F$ be a skew-commutative connected (i.e. $F_{0}=A$ ) $D G A$-algebra. Then there exist a free skew-commutative $D G R$-algebra $U$ and a surjective homomorphism $u$ : $U \rightarrow F$ of $D G$ rings, such that $u$ coincides with $u_{0}$ in dimension zero and $H(u)$ is an isomorphism. If, moreover, $F_{i}$ is a finitely generated $A$-module for each $i$, and $R$ is noetherian, then $U$ can be chosen to be locally (i.e. in each degree) finitely generated.

Proof. Setting $u(-1)=u_{0}$ and $U(-1)=R$, we can assume by induction that a homomorphism of DG rings $u(i): U(i) \rightarrow F$ has been constructed, which is surjective in degrees $\leqslant i$, and induces an epimorphism $Z_{i}(u(i)): Z_{i}(U(i)) \rightarrow Z_{i}(F)$ and isomorphisms $H_{j}(u(i))$ for $j<i$. By standard diagram chasing we can find free $R$-modules $T_{i+1}^{\prime}, T_{i+1}^{\prime \prime}$, and $T_{i+1}$, and dotted maps, such that the diagrams of $R$-modules depicted below commute and satisfy the following conditions:

$$
\begin{aligned}
& \operatorname{Im}\left(p \delta^{\prime}\right)=\operatorname{Ker} H_{i}(u(i)), \\
& \operatorname{Im}\left(u_{i+1}^{\prime \prime}\right)=Z_{i+1}(F), \\
& \operatorname{Im}\left(u_{i+2}\right)=F_{i+2}
\end{aligned}
$$

(of course, all $A$-modules are viewed as $R$-modules via $u_{0}$ ).

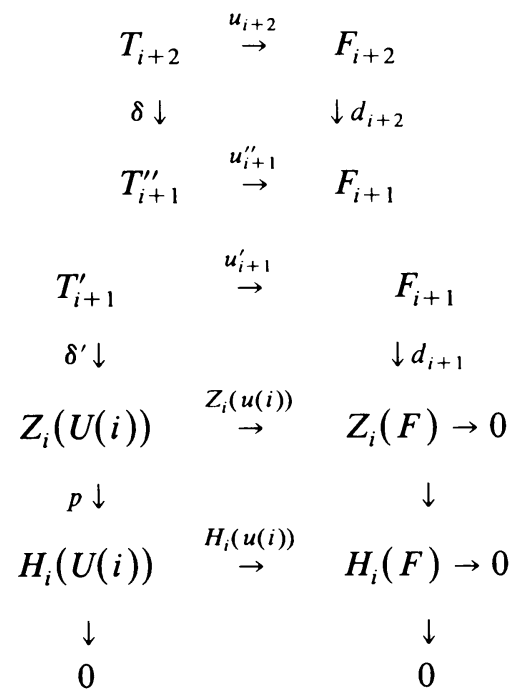


Assigning to $T_{i+1}^{\prime} \oplus T_{i+1}^{\prime \prime}$ degree $i+1$, to $T_{i+2}$ degree $i+2$, and denoting by $V(\quad$ ) the free skew-commutative algebra functor, we set

$$
\begin{array}{ccrl}
U(i+1)=U(i) \otimes V\left(T_{i+1}^{\prime} \oplus T_{i+1}^{\prime \prime}\right) \otimes V\left(T_{i+2}\right), \\
u(i+1) \mid U(i)=u(i), & & d \mid U(i)=d, \\
u(i+1) \mid T_{i+1}^{\prime}=u_{i+1}^{\prime}, & & d \mid T_{i+1}^{\prime}=\delta^{\prime}, \\
u(i+1) \mid T_{i+1}^{\prime \prime}=u_{i+1}^{\prime \prime}, & & d \mid T_{i+1}^{\prime \prime}=0, \\
u(i+1) \mid T_{i+2}=u_{i+2}, & & d \mid T_{i+2}=\delta .
\end{array}
$$

These conditions completely define the DG $R$-algebra $U(i+1)$ and the homomorphism of DG rings $u(i+1)$. By construction, $u(i+1)$ is surjective in degrees $\leqslant i+1$, and induces an epimorphism $Z_{i+1}(u(i+1))$ and isomorphisms $H_{j}(u(i+1))$ for $j<i+1$. Of course, with $U=\lim U(i)$ and $u=\lim u(i)$, we have established our claim. The finiteness condition is achieved by choosing finitely generated $T_{i}$ 's.

Proposition 2.3. Suppose $F$ satisfies condition (1.3)(i). Then $F$ has a free locally finitely generated $k$-model.

If $S$ is a set of elements of $I H(F)$, which admits a trivial Massey operation, then the set $\alpha(S) \subset I H(V)$ admits a trivial Massey operation.

Proof. Set $F=F \otimes_{A} \hat{A}$, where $\hat{A}$ denotes the m-adic completion of $A$. Choose, by Cohen's structure theorem for complete local rings, a regular local ring $R$ with $\operatorname{edim} R=\operatorname{edim} \hat{A}$ and a surjective ring homomorphism $u_{0}: R \rightarrow \hat{A}$. Let $u: U \rightarrow F$ be the homomorphism constructed in the lemma, with $U$ free skew-commutative and locally finitely generated. Let $S_{1}, \ldots, S_{n}$ be elements of $U_{1}$ whose images under $u_{0} d_{1}$ form a minimal set of generators of $m$. Then the $S_{i}$ 's form part of a basis of the free $R$-module $U_{1}$. Denote by $J$ the DG ideal of $U$ generated by $S_{1}, \ldots, S_{n}, d S_{1}, \ldots, d S_{n}$. The argument used to prove [AG, Proposition 1] shows, with an easy induction on $n$, that $H_{i}(J)=0$ for all integers $i$. Setting $V=U / J$, we see that the natural projection from $U$ to $V$ induces an isomorphism in homology. Since $J$ contains the maximal ideal of $R, V$ is a locally finitely generated free $k$-algebra and, hence, the required model.

For the second statement of the proposition, it is clearly sufficient to establish for a homomorphism of DG rings $w: Y \rightarrow Y^{\prime}$, such that $H(w)$ is an isomorphism, that (a) if $S \subset I H(Y)$ admits a t.M.o. $\gamma$, then $S^{\prime}=H(w)(S)$ admits a t.M.o. $\gamma^{\prime}$; and (b) the converse statement, assuming that $w$ is surjective. Part (a) is straightforward, since $\gamma^{\prime}=w \gamma$ is a t.M.o. For (b), writing $W$ for $\operatorname{Ker}(w)$, we have the exact sequence

$$
0 \rightarrow B(W) \rightarrow Z(Y) \stackrel{Z(w)}{\rightarrow} Z\left(Y^{\prime}\right) \rightarrow 0 .
$$

Hence we can lift $\gamma^{\prime}$ to a $\gamma$ defined on the elements (i.e. on sequences of length one) of $S=H(w)^{-1} S^{\prime}$. By induction we can assume that $\gamma$ has been defined on sequences of elements of $S$ of length $<n$ in such a way that $w \gamma\left(f_{1}, \ldots, f_{m}\right)=\gamma^{\prime}\left(w_{*} f_{1}, \ldots, w_{*} f_{m}\right)$ 
$\left(w_{*}=H(w)\right)$. If $y$ is any element of $Y$ mapping onto $\gamma^{\prime}\left(w_{*} f_{1}, \ldots, w_{*} f_{n}\right)$ then

$$
\begin{aligned}
w(d y) & =d \gamma^{\prime}\left(w_{*} f_{1}, \ldots, w_{*} f_{n}\right) \\
& =\sum_{i=1}^{n-1} \bar{\gamma}^{\prime}\left(w_{*} f_{1}, \ldots, w_{*} f_{i}\right) \gamma^{\prime}\left(w_{*} f_{i+1}, \ldots, w_{*} f_{n}\right) \\
& =w\left(\sum_{i=1}^{n-1} \bar{\gamma}\left(f_{1}, \ldots, f_{i}\right) \gamma\left(f_{i+1}, \ldots, f_{n}\right)\right) .
\end{aligned}
$$

It follows from the exact sequence that there exists an $x \in Y$ such that

$$
\sum_{i=1}^{n-1} \bar{\gamma}\left(f_{1}, \ldots, f_{i}\right) \gamma\left(f_{i+1}, \ldots, f_{n}\right)-d y=d x
$$

hence we can take $\gamma\left(f_{1}, \ldots, f_{n}\right)=x+y$.

In order to fix notations we recall

Definition 2.4 (cf. [Cl,(3.1); M,(1.4)]). A s.h.m. (= strongly homotopy multiplicative) map $h: G \rightarrow V$ of DG $k$-algebras is a collection of $k$-linear maps $\left\{h_{n}\right.$ : $\left.(I G)^{\otimes n} \rightarrow I V\right\}_{n \geqslant 1}$ such that $h_{n}$ has degree $n-1$ and for each $n$ the following identity is satisfied:

$$
\begin{aligned}
d h_{n}\left(g_{1}, \ldots, g_{n}\right)= & \sum_{j=1}^{n} h_{n}\left(\bar{g}_{1}, \ldots, \bar{g}_{j-1}, d g_{j}, g_{j+1}, \ldots, g_{n}\right) \\
& -\sum_{j=1}^{n-1} h_{n-1}\left(\bar{g}_{1}, \ldots, \bar{g}_{j-1}, \bar{g}_{j} g_{j+1}, g_{j+2}, \ldots, g_{n}\right) \\
& +\sum_{j=1}^{n-1} h_{j}\left(\bar{g}_{1}, \ldots, \bar{g}_{j}\right) h_{n-j}\left(g_{j+1}, \ldots, g_{n}\right) .
\end{aligned}
$$

Observation 2.5. Let $V$ be a DG algebra over $k$ and let $\left\{v_{i}\right\}_{i \in I}$ be a set of elements of $I Z(V)$. Let $G$ be the connected DG $k$-algebra defined up to (a noncanonical) isomorphism by the following conditions:

(i) $I G$ has a basis $\left\{g_{i}\right\}_{i \in I}$ such that $\operatorname{deg} g_{i}=\operatorname{deg} x_{i}$;

(ii) $(I G)^{2}=0$;

(iii) $d G=0$.

Then $S=\left\{\left[v_{i}\right]\right\}_{i \in I}$ admits a t.M.o. if and only if there exists a s.h.m. map $h: G \rightarrow V$ such that $h_{1}\left(g_{i}\right)=v_{i}(i \in I)$.

Indeed, for a given $h$ define $\gamma\left(\left[v_{1}\right], \ldots,\left[v_{n}\right]\right)=h_{n}\left(g_{1}, \ldots, g_{n}\right)$. Conversely, given a t.M.o. $\gamma$, set $h_{n}\left(g_{1}, \ldots, g_{n}\right)=\gamma\left(\left[v_{1}\right], \ldots,\left[v_{n}\right]\right)$.

In part $\mathrm{C}$ of the proof we are going to apply this observation through the following result of Clark.

LEMMA 2.6 [Cl,(3.2)]. If $h: G \rightarrow V$ is a s.h.m. map, it induces a homomorphism $\bar{B}(h): \bar{B}(G) \rightarrow \bar{B}(V)$ of $D G$ coalgebras over $k$, where $\bar{B}(\quad)$ denotes the reduced bar-construction with its standard diagonal map.

B. Divided powers for DG torsion functors. We shall construct such a structure only in the special case of algebras satisfying the conditions (1.3); their existence in 
general for connected algebras is an interesting open question. For the general properties of the Eilenberg-Moore DG torsion functors we refer to [GM], and for the Hopf algebra structure they carry in the cases considered here, to [Av 1].

Lemma 2.7. Suppose $F$ satisfies conditions (1.3). Then $T^{F}=\operatorname{Tor}^{F}(k, k)$ has a unique structure of Hopf $\Gamma$-algebra for which the map $T^{\eta}=\operatorname{Tor}^{\eta}(k, k)$ induced by the structure map $\eta: A \rightarrow F$ is a homomorpism of Hopf $\Gamma$-algebras. Moreover, $T^{\eta}$ is surjective.

Proof. It is shown in $[A v 1,(1.1)]$ that $T^{F}$ has a natural structure of Hopf algebra. Furthermore, it is proved in [Av 2,(2.2)], that $T^{\eta}$ is a surjective homomorphism of Hopf algebras with kernel equal to $I(k \otimes F) \cdot T^{A}$. This ideal is closed under the divided powers of $T^{A}$ by our assumption (1.3)(iii), and the claim follows.

In particular, as for Theorem 0.3 , one can apply the result of [An] and [Sj] to see that the graded dual $E_{F}$ of $T^{F}$ is as a Hopf algebra the universal enveloping algebra of its Lie subalgebra formed by the elements orthogonal to $T_{0}^{F} \oplus I^{(2)} T^{F}$. We shall denote this Lie algebra by $L_{F}$.

Next we recall a definition from [Av 2,(2.4)]. For $z \in I Z(F)$ choose $y \in X$ such that $d y=-z$. Then $[z] \mapsto 1 \otimes y$ defines a degree one $k$-linear map $\sigma_{F}: I H(F) \rightarrow T^{F}$ called the suspension. It appears in the statement of the following proposition, which has been proved also by M. André.

Proposition 2.8. The kernel of the composite map

$$
I H(F) \stackrel{\sigma_{F}}{\rightarrow} T^{F} \stackrel{\pi_{F}}{\rightarrow} T^{F} / I^{(2)} T^{F}
$$

is the set $M H(F)$ of all elements of $I H(F)$ decomposable as matric Massey products.

Proof. By an application of some results of Gugenheim and May, it was shown in $[A v 2,(2.6)]$ that $\operatorname{Ker} \sigma_{F}=M H(F)$. Hence we have to show that $\operatorname{Im} \sigma_{F} \cap I^{(2)} T^{F}=0$. Let $P\left(T^{F}\right)$ denote the subspace of primitive elements of the Hopf algebra $T^{F}$. By [An, Proposition 8], one knows that the restriction of $\pi_{F}$ to $P\left(T^{F}\right)$ is injective. Hence it suffices to show that $\operatorname{Im} \sigma_{F} \subset P\left(T^{F}\right)$. From the definition of $\sigma_{F}$, given in [GM], $\operatorname{Im} \sigma_{F} \subset I_{1}$, where $0 \subset I_{0}=T_{0}^{F} \subset I_{1} \subset \cdots$ is the increasing filtration of $T^{F}$ defined by the Eilenberg-Moore spectral sequence with $E_{p, q}^{2}=\operatorname{Tor}_{p, q}^{H(F)}(k, k) \Rightarrow$ $\operatorname{Tor}_{p+q}^{F}(k, k)$. This is a sequence of Hopf algebras [Av 1], hence the term $E^{\infty}=$ $\bigoplus_{j \geqslant 0} I_{j} / I_{j-1}$ is a bigraded Hopf algebra associated to $T^{F}$. But the elements of $E_{1, *}^{\infty}=I_{1} / I_{0}$ are primitive for dimensional reasons. It follows that the positive dimensional elements of $I_{1}$ are primitive in $T^{F}$, which implies $\operatorname{Im} \sigma_{F} \subset P\left(T^{F}\right)$.

C. End of the proof. Let $V$ be a $k$-model of $F$ as in Proposition 2.3. Denote by $\alpha$ the isomorphism $I H(F) \simeq I H(V)$ realized by the chosen homotopy equivalence of $F$ to $V$, and by $\beta$ the corresponding homomorphism of DG torsion functors; note that $\beta$ is an isomorphism of Hopf algebras. With the notations of Theorem 1.4, choose $v_{i} \in I Z(V)$ with $\left[v_{i}\right]=\alpha\left(f_{i}\right)(i \in I)$. By Proposition 2.3, $\left\{\left[v_{i}\right]\right\}_{i \in I}$ admit a t.M.o., hence as observed in (2.5) there is a s.h.m. map $h: G \rightarrow V$ with $h_{1}\left(g_{i}\right)=v_{i}$. Now 
consider the diagram, all of whose maps have already been defined:

$$
\begin{array}{ccccccc}
I G & \stackrel{I H\left(h_{1}\right)}{\rightarrow} & I H(V) & \stackrel{\alpha}{\leftarrow} & I H(F) & & \\
\sigma_{G} \downarrow & & \sigma_{V} \downarrow & & \sigma_{F} \downarrow & & \\
\bar{B}(G) \stackrel{H \bar{B}(h)}{\rightarrow} & \operatorname{Tor}^{V}(k, k) & \stackrel{\beta}{\leftarrow} & T^{F} & \stackrel{T^{\eta}}{\leftarrow} & T^{A} \\
& & & & \pi_{F} \downarrow & & \downarrow \pi_{A} \\
& & & & Q\left(T^{F}\right) & \stackrel{Q\left(T^{\eta}\right)}{\leftarrow} & Q\left(T^{A}\right) .
\end{array}
$$

The commutativity of the lower square follows from the fact that $T^{\eta}$ is a map of $\Gamma$-algebras over $k$ (Lemma 2.7), that of the two upper squares is a consequence of the naturality of the suspension [Av 2, (2.5)]. Set $h_{*}=H \bar{B}(h)$.

Claim. The composite map $\delta=\pi_{F} \beta^{-1} h_{*} \sigma_{G}$ is injective.

First note that by [M, (1.5)], $\alpha M H(F)=M H(V)$, hence condition (2) of Theorem 1.4 together with Gugenheim's and May's evaluation of the kernel of the suspension show that $\operatorname{Im} I H\left(h_{1}\right) \cap \operatorname{Ker} \sigma_{V}=0$. This implies that $h_{*}$ is injective on $\operatorname{Im} \sigma_{G} \simeq I G$. But $\operatorname{Im} \sigma_{G}$ is the set of primitive elements of $\bar{B}(G) ; h_{*}$ being a homomorphism of coalgebras (Lemma 2.6), which is injective on the primitives, is injective globally. Having proved that $\beta^{-1} h_{*} \sigma_{G}$ is injective, we see that $\operatorname{Im}\left(\alpha^{-1} \circ I H\left(h_{1}\right)\right)$ intersects Ker $\sigma_{F}=M H(F)$ trivially, and the claim follows from Proposition 2.8.

After dualization, the relevant information obtained so far can be summarized in the commutative diagram

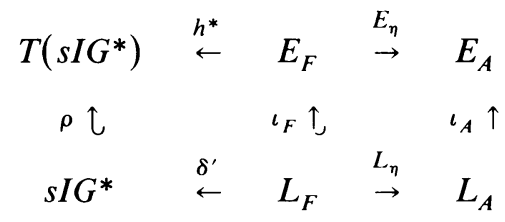

in which:

$T($ ) denotes the tensor algebra functor;

$\rho$ is the natural injection of the tensors of length one;

$h^{*}$ is a surjective homomorphism of algebras;

$E_{\eta}$ is an injective homomorphism of Hopf algebras;

$L_{\eta}$ is an injective homomorphism of Lie algebras;

$\iota_{F}$ and $\iota_{A}$ are the injections of the corresponding Lie algebras in their universal envelopes;

$\delta^{\prime}$ is the degree zero $k$-linear map, obtained by composing $\delta^{*}$ with the degree one isomorphism of $I G^{*}$ to $s\left(I G^{*}\right) ; \delta^{\prime}$ is surjective by the claim established above.

Let $\varepsilon$ be a splitting of $\delta^{\prime}$. By the universal property of the tensor algebra, there is a unique algebra homomorphism $\nu: T\left(s I G^{*}\right) \rightarrow E_{F}$, whose restriction to the tensors of length one coincides with $\iota_{F} \circ \varepsilon$. The map $\nu$ is injective, since it is a splitting of $h^{*}$, and it sends into $L_{F}$ the free Lie algebra, generated by $\rho\left(s I G^{*}\right)$ in $T\left(s I G^{*}\right)$ under the commutation operation. The image of this Lie algebra under $L_{\eta} \circ \nu$ is the required Lie subalgebra of $L_{A}$. 
D. Proof of Corollary 1.5. Let $R=k \llbracket X_{1}, \ldots, X_{r} \rrbracket$ be the power series ring, and let $M_{1}, \ldots, M_{s}$ denote the monomials in the indeterminates, corresponding to the monomials $m_{1}, \ldots, m_{s}$ in the $x_{i}$ 's. By [Av 2,(6.2)] there is an isomorphism of Hopf algebras

$$
\operatorname{Tor}^{A}(k, k) \otimes_{\operatorname{Tor}^{B}(k, k)} k \simeq \operatorname{Tor}^{S}(k, k) \otimes_{\operatorname{Tor}^{R}(k, k)} k
$$

(where $S=R /\left(M_{1}, \ldots, M_{s}\right)$ ), which can be shown, using Lemma 2.7, to commute with the divided powers. Since both homomorphisms $B \rightarrow A$ and $R \rightarrow S$ induce injective maps in homology (loc. cit.) it follows from the above isomorphism and [Av 2, (1.3)] that $\operatorname{Ker}\left(L_{A} \rightarrow L_{B}\right) \simeq \operatorname{Ker}\left(L_{S} \rightarrow L_{R}\right)$ as Lie algebras; hence it suffices to prove the statement for $A=S$. In this case if $\operatorname{grade}\left(M_{1}, \ldots, M_{s}\right) \neq s, S$ is not a complete intersection, and two of the monomials are divisible by one of the indeterminates. The corresponding one-dimensional homology classes in the homology of the Koszul complex of $S$ satisfy the conditions of Remark 1.7(2)(a), hence Theorem 1.4 applies.

\section{Proof of Theorem 1.1.}

A. Preliminaries on the Koszul complex. We shall denote by $K^{A}$ or simply by $K$ the Koszul complex on a minimal set of generators of $m$. It is well known that $H(K)$ is up to isomorphism independent of the choice of the generators. For ease of reference we collect several known results.

LEMMA 3.0. For every local ring $A$,

$$
e_{2}(A)=\operatorname{dim}_{k} H_{1}(K), \quad e_{3}(A)=\operatorname{dim}_{k} H_{2}(K) / H_{1}^{2}(K) .
$$

Lemma 3.1 [AG]. If depth $A=d$, there exists a minimal set $t_{1}, \ldots, t_{n}$ of generators of $\mathrm{m}$ such that $t_{1}, \ldots, t_{d}$ form a regular sequence, and the epimorphism from the Koszul complex on $t_{1}, \ldots, t_{n}$ to the Koszul complex on the images of $t_{d+1}, \ldots, t_{n}$ in $\bar{A}=$ $A /\left(t_{1}, \ldots, t_{d}\right)$ induces an isomorphism $H\left(K^{A}\right) \stackrel{\sim}{\rightarrow} H\left(K^{\bar{A}}\right)$.

LEMMA 3.2. The following are equivalent:

(1) $A$ is a complete intersection;

(2) $H(K)$ is the exterior algebra on $H_{1}(K)$;

(3) $H_{2}(K)=H_{1}^{2}(K)$;

(4) $\left(H_{1}(K)\right)^{\operatorname{edim} A-\operatorname{depth} A} \neq 0$.

$((1) \Rightarrow(2)$ is due to Tate, $(3) \Rightarrow(1)$ is due to Assmus; for proofs cf. [GL]; (4) $\Rightarrow(1)$ is Wiebe's main result in [W].)

LEMMA 3.3 [AG]. The following are equivalent:

(1) A is Gorenstein;

(2) there exists an $n \geqslant 0$ such that the natural pairings (induced by the multiplication in $K) H_{i}(K) \times H_{n-i}(K) \rightarrow H_{n}(K)$ are perfect for all $i \geqslant 0$.

When these conditions are satisfied, one has $n=\operatorname{edim} A-\operatorname{depth} A$, and $H_{n}(K) \simeq$ $k$. 
Lemma 3.4 [Av 3]. Let $A$ be a Cohen-Macaulay almost complete intersection (i.e. $d(A)=1)$ with $\operatorname{edim} A-\operatorname{depth} A=n$. Then $e_{3}(A)=\operatorname{dim}_{k} H_{n}(K)$.

LEMma 3.5. Suppose that one of the following conditions is fulfilled:

(a) $\operatorname{edim} A-\operatorname{dim} A \leqslant 1$; or

(b) $\operatorname{edim} A-\operatorname{depth} A \leqslant 2$.

Then $A$ is either a complete intersection or a Golod ring. Moreover, Golod rings satisfying (b) have $e_{3}(A)=e_{2}(A)-1$.

Condition (a) is treated by Shamash [Sh], while (b) is settled by Scheja [Sc]; note that a direct proof of Scheja's result can be obtained along the lines of Lemma 3.8 below.

In order to prove Theorem 1.1 we shall use a specialization of Theorem 1.4, which we now state explicitly.

Proposition 3.6. (a) Let $\operatorname{edim} A-\operatorname{depth} A \leqslant 3$. Suppose $L \subset I H(K)$ is a graded subspace such that:

(1) $L^{2}=0$; and

(2) $L \cap I^{2} H(K)=0$.

Then $L_{A}$ contains a Lie subalgebra isomorphic to the free graded Lie algebra on $s L^{*}$.

(b) Let $A$ be Gorenstein with $\operatorname{edim} A-\operatorname{dim} A=4$. Suppose there exist linearly independent $f_{1}, f_{2} \in H_{1}(K)$ such that $f_{1} \cdot f_{2}=0$. Then $L_{A}$ contains a free graded Lie subalgebra on two generators of degree 2.

Proof. Clearly $F=K$ satisfies conditions (1.3). Choose $\bar{A}$ as in Lemma 3.1. Suppose we have proved that a t.M.o. can be defined on the image in $H\left(K^{\bar{A}}\right)$ of a basis of $L$ (resp. of $f_{1}$ and $f_{2}$ ). Then by Lemma 3.1 and by (the proof of) Proposition 2.3, a t.M.o. can be defined on a basis of $L$ (resp. on $\left\{f_{1}, f_{2}\right\}$ ), hence condition (1) of Theorem 1.4 is satisfied by this set. Since the second condition of the theorem is satisfied in view of our assumption (2) and Remark 1.7(3), we get our result as a special case of the theorem.

It remains to prove that a t.M.o. can be defined on a basis $f_{1}, \ldots, f_{m}$ of $L$ (resp. on $\left.\left\{f_{1}, f_{2}\right\}\right)$, assuming depth $A=0$. In case (a) choose a set $z_{1}, \ldots, z_{m}$ of cycles such that $\left[z_{i}\right]=f_{i}$. According to our condition (1) there exist $y_{i j} \in I K$ such that $d y_{i j}=z_{i} z_{j}$. Set $\gamma\left(f_{i}\right)=z_{i}, \gamma\left(f_{i}, f_{j}\right)=y_{i j}$, and $\gamma\left(f_{i_{1}}, \ldots, f_{i_{1}}\right)=0$ for $l>2$. For dimensional reasons it is clear that $\gamma$ is a t.M.o. on $\left\{f_{1}, \ldots, f_{m}\right\}$ since $K_{i}=0$ for $i>3$.

In case (b) choose $y \in K_{3}$ such that $d y=z_{1} z_{2}\left(\left[z_{i}\right]=f_{i}\right)$. Since $A$ is artinian of embedding dimension $4, Z_{4}(K)=H_{4}(K)$ is one dimensional over $k$ generated say by $e$. By Poincaré duality (Lemma 3.3), one can choose $u_{1}, u_{2} \in Z_{3}(K)$ such that $f_{i} \cdot\left[u_{j}\right]=\delta_{i j} e(i, j \in\{1,2\})$; in particular, this means that $z_{1} u_{2}=0=z_{2} u_{1}$. Let $z_{i} y=a_{i} e$ with $a_{i} \in k(i=1,2)$. Set $\gamma\left(f_{i}\right)=z_{i}, \gamma\left(f_{1}, f_{2}\right)=-\gamma\left(f_{2}, f_{1}\right)=y-a_{1} u_{1}-$ $a_{2} u_{2}$, and $\gamma=0$ on all other sequences of $f_{1}, f_{2}$. Since $z_{i} \gamma\left(f_{1}, f_{2}\right)=0$, it is clear that $\gamma$ is a t.M.o. on $\left\{f_{1}, f_{2}\right\}$.

B. edim $A-\operatorname{depth} A \leqslant 2$. Assume that $A$ is not a complete intersection. Then $e_{2}(A)>1$, and by Lemma 3.5 (b) $A$ is a Golod ring. By Corollary 1.6 or by Proposition 3.6(a) $L_{A}$ contains a free Lie subalgebra on $\operatorname{sIH}(K)^{*}$ which proves the theorem for this class. 
C. edim $A-\operatorname{depth} A=3$. In order to apply Proposition 3.6 in this case we have first to establish some results on the structure of $H(K)$.

Lemma 3.7. Suppose $A$ satisfies condition $\mathrm{C}$ and that $\operatorname{edim} A-\operatorname{dim} A=2$. Then $e_{3}(A) \geqslant e_{2}(A)-2$.

Proof. By Lemmas 3.1 and 3.0 we can assume $e_{1}(A)=\operatorname{edim} A=3, \operatorname{dim} A=1$, $\operatorname{depth} A=0$. Let $\mathfrak{p}$ be a nonmaximal prime of $\hat{A}$. In the notation of the introduction, $\chi_{3}(A)=\chi_{3}(\hat{A})$. On the other hand, by Gulliksen's theorem [Gu, (3.3)] (i.e., Conjecture $\mathrm{C}_{5}$ for $r=3$ and complete rings), we have

$$
\chi_{3}(\hat{A}) \geqslant \chi_{3}\left(\hat{A}_{\mathfrak{p}}\right)+\operatorname{dim} \hat{A} / \mathfrak{p}
$$

hence

$$
e_{3}(A) \geqslant e_{2}(A)-2+\chi_{3}\left(\hat{A}_{\mathfrak{p}}\right) .
$$

By Lech's theorem [L, Theorem 3] (i.e. Conjecture $\mathrm{C}_{5}$ for $r=1$, which is easily checked directly for complete rings), we see that $e_{1}\left(\hat{A}_{\mathfrak{p}}\right) \leqslant e_{1}(\hat{A})-\operatorname{dim} \hat{A} / \mathfrak{p} \leqslant 2$. It follows from Lemma 3.5(b) that the artinian ring $\hat{A}_{\mathrm{p}}$ is either a complete intersection, in which case $\chi_{3}\left(\hat{A}_{\mathfrak{p}}\right)=0$, or is a Golod ring, with $\chi_{3}\left(\hat{A}_{\mathfrak{p}}\right)=2-e_{2}\left(\hat{A}_{\mathfrak{p}}\right)+\left(e_{2}\left(\hat{A}_{\mathfrak{p}}\right)\right.$ $-1)=1$. This proves our claim.

Lemma 3.8. Suppose $A$ is a Cohen-Macaulay ring satisfying condition $\mathrm{C}$. Then either $A$ is a complete intersection, or $H_{1}(K)$ contains linearly independent elements $f_{1}, f_{2}$, such that $f_{1} \cdot f_{2}=0$.

Proof. By Lemma 3.1 we can assume $A$ is artinian, hence $A=R / \mathfrak{a}$ with $(R, \mathfrak{n})$ regular of dimension three and $a \mathfrak{n}$-primary with $a \subset \mathfrak{n}^{2}$. Choose a minimal set of generators $x_{1}, \ldots, x_{r}$ of a such that $x_{1}, x_{2}, x_{3}$ form an $R$-regular sequence. If $A$ is not a complete intersection, the ring homomorphism $R /\left(x_{1}, \ldots, x_{4}\right) \rightarrow A$ induces a homomorphism of Koszul homology algebras which is injective in degree one by the minimality of the chosen set of generators. Hence it is sufficient (and necessary) to prove the lemma for almost complete intersections. Setting $t=\operatorname{dim}_{k} H_{3}(K)$, we have $\operatorname{dim}_{k} H_{2}(K)=t+4-1=t+3$. From Lemma 3.4 we have $\operatorname{dim}_{k} H_{1}^{2}(K)=3$. Our claim now follows from the next general statement.

LEMMA 3.9. Let $A$ be an arbitrary local ring, with $\operatorname{dim}_{k} H_{1}(K)=r$. If $\operatorname{dim}_{k} H_{1}^{2}(K)$ $\leqslant 2(r-2)$, then there exist linearly independent $f_{1}, f_{2} \in H_{1}(K)$ such that $f_{1} \cdot f_{2}=0$.

Proof. Consider the kernel $M$ of the canonical epimorphism $\Lambda^{2} H_{1}(K) \rightarrow H_{1}^{2}(K)$. Our assumption means that the linear variety defined by $M$ in $\mathbf{P}=\mathbf{P}_{k}\left(\Lambda^{2} H_{1}(K)\right)$ is of codimension $\leqslant 2(r-2)$. Hence it has a nonempty intersection with the $2(r-2)$-dimensional Grassmannian of projective lines in $\mathbf{P}_{k}\left(H_{1}(K)\right)$ embedded in $\mathbf{P}$ by the Plücker embedding. This implies the assertion of the lemma.

Remark. In fact, the algebra $H(K)$ for Cohen-Macaulay almost complete intersections of embedding codimension 3 has been explicitly computed in [Go 2] and [Av 3]. 
We now turn to the proof of Theorem 1.1 assuming condition $\mathrm{C}$. Note that it implies the inequality edim $A-\operatorname{dim} A \leqslant 3$. When this difference does not exceed 1 , we are done by applying Lemma 3.5(a) and Corollary 1.6.

Assume edim $A-\operatorname{dim} A=2$, and let $L^{\prime}$ be the vector space generated by some lifting to $H_{2}(K)$ of a $k$-basis of $H_{2}(K) / H_{1}^{2}(K)$. Clearly $L=L^{\prime}$ satisfies the conditions of Proposition 3.6(a), hence our claim follows from Lemma 3.7 when $e_{2}(A)>3$. The algebras $H(K)$ for noncomplete intersections with $e_{2}(A)=3$ have been computed in [Av 2, (7.2)]. In particular, it is shown there that either $H_{1} H_{2}=0$, or $A=A^{\prime} /(x)$, where $\left(A^{\prime}, \mathrm{m}^{\prime}\right)$ is a Golod ring with edim $A^{\prime}-\operatorname{depth} A^{\prime}=2$, and $x$ is a nonzero divisor contained in $\mathrm{m}^{\prime 2}$. In the second case $H(K) \simeq H\left(K^{A^{\prime}}\right) \otimes_{k} k[f]$, $\operatorname{deg} f=1, f^{2}=0$, hence $L=\operatorname{Im}\left(I H\left(K^{A^{\prime}}\right) \rightarrow I H(K)\right)$ satisfies, according to Lemma 3.5(b), the conditions of Proposition 3.6(a). In the first case we take $L=L^{\prime} \oplus H_{3}(K)$, and note that $H_{3}(K) \neq 0$ by the assumption of depth zero, while $L^{\prime} \neq 0$ by Lemma 3.2 .

Finally let $\operatorname{edim} A-\operatorname{dim} A=3$, i.e. let $A$ be a Cohen-Macaulay ring. Then the elements $f_{1}, f_{2}$ of Lemma 3.8 generate a two-dimensional subspace $L$ satisfying the conditions of $3.6(\mathrm{~b})$.

D. edim $A-\operatorname{dim} A=4$, A Gorenstein. The following lemma, shown to us by $\mathrm{J}$. Backelin, and independently proved by A. Kustin and M. Miller, gives a shortcut to the original treatment of this case.

Lemma 3.10. Suppose $A$ satisfies condition D. Then either $A$ is a complete intersection (i.e. $e_{3}(A)=0$ ), or $e_{3}(A) \geqslant e_{2}(A)-1$.

Proof. Assume $0<e_{3}(A)<e_{2}(A)-1=r-1$. Since $H(K)$ is a Poincaré algebra (Lemma 3.3), and $H_{i}(K)=0$ for $i>4, \operatorname{dim}_{k} H_{2}(K)=2(r-1)$, hence $\operatorname{dim}_{k} H_{1}^{2}(K)=\operatorname{dim}_{k} H_{2}(K)-e_{3}(A) \geqslant r$. Denoting by ${ }^{\perp}$ orthogonal complements with respect to the nondegenerate bilinear form $H_{2}(K) \times H_{2}(K) \rightarrow H_{4}(K) \simeq k$, which is symmetric if char $k \neq 2$ and alternating otherwise (cf. [Av 2, (9.4)]), we get $\operatorname{dim}_{k}\left(H_{1}^{2}(K)\right)^{\perp} \leqslant r-2$. It follows that $H_{1}^{2}(K) \not \subset\left(H_{1}^{2}(K)\right)^{\perp}$, hence $H_{1}^{4}(K) \neq 0$, which contradicts Wiebe's result; cf. Lemma 3.2.

Now let $A$ be a noncomplete intersection satisfying condition D. By the lemma $\operatorname{dim}_{k} H_{1}^{2}(K)=\operatorname{dim}_{k} H_{2}(K)-e_{3}(A) \leqslant r-1$, and this integer is $\leqslant 2(r-2)$ since $r>4$. Lemma 3.9 shows that the conditions of Proposition 3.6(b) are satisfied, and this finishes the proof of the theorem.

4. Proof of Theorem 1.2. Suppose that $A$ satisfies the conditions of the theorem, and is not a complete intersection. The equivalence of (i), (ii), and (vi) is a consequence of Theorem 1.1 and Proposition 0.7. If, moreover, $A$ is Cohen-Macaulay, it has been shown in Lemmas 3.5(b), 3.8 and 3.10 that $H_{1}(K)$ contains two linearly independent elements admitting a t.M.o., hence $L_{A}$ contains a free Lie subalgebra generated by two elements of degree 2 . The same conclusion holds also under the assumption edim $A-\operatorname{dim} A=1$ by Lemma 3.5(a). It follows that in these cases $e_{2 i}(A) \neq 0$ for all $i>0$, and also that $r_{A} \leqslant \sqrt{2} / 2$, the last inequality being a consequence of the relation $P_{A}(t) \gg\left(1-2 t^{2}\right)^{-1}$. 
In order to finish the proof we have still to treat the rings satisfying the conditions

$$
\operatorname{edim} A-\operatorname{depth} A=3, \quad \operatorname{edim} A-\operatorname{dim} A=2 .
$$

We want to prove for them the relations:

(iii) ${ }^{\circ} r_{A} \leqslant \sqrt{2} / 2$; and

(iv) $e_{6 i} \neq 0$ for $i>0$.

From Lemma 3.7 and Proposition 3.6(a) $L_{A}$ contains a free graded Lie subalgebra generated by $e_{2}(A)-2$ elements of degree 3 . As in the proof of the implication $\mathrm{C}_{10} \Rightarrow \mathrm{C}_{2}$ of Proposition 0.7 (cf. $\$ 5$ ), it follows that it also contains a free graded Lie subalgebra on $e_{2}(A)-2$ generators of degree 6 . Hence for $e_{2}(A) \geqslant 4$, (iv) ${ }^{\circ}$ is established, while in the remaining case there is the more precise

Lemma 4.2. Suppose $A$ satisfies (4.1) and has $e_{2}=3$. Then $f_{i}(A)>0$ for $i \geqslant 2$ (cf. Conjecture $\mathrm{C}_{3}{ }^{\prime}$ in the introduction).

Proof. We use the technique and notation developed by Gulliksen [Gu, §4]: $\varphi$ denotes the well-known isomorphism from the multiplicative group $1+t \mathbf{Z} \llbracket t \rrbracket$ to the additive group $t \mathbf{Z} \llbracket t \rrbracket$, which in particular sends $1 / G(t)=P_{A}(t) /(1+t)^{e_{1}(A)}$ to $-\sum_{i \geqslant 0} f_{i}(A) t^{i}$. According to [Av 2,(7.2)], the following expressions for $G(t)$ can occur:

$$
\begin{gathered}
\left(1-t^{2}\right)\left(1-2 t^{2}-t^{3}\right) \\
1-3 t^{2}-(m-s+2) t^{3}-(m-s) t^{4} \\
(m, s)=(1,0),(1,1), \text { or } m>1,0 \leqslant s \leqslant 2 \\
1-3 t^{2}-(m-1) t^{3}-(m-3) t^{4}-t^{5}-t^{6}, \quad m>1,
\end{gathered}
$$

where $m=\operatorname{dim}_{k} H_{3}(K), s=\operatorname{dim}_{k} H_{1}^{2}(K)$. In order to establish the inequality $\varphi(G(t)) \gg t^{2}(1-t)^{-1}$, we use the inequality $\varphi\left(1-2 t^{2}-t^{3}\right) \gg t^{2}(1-t)^{-1}$ obtained in the proof of [Gu, (4.1)(i)]. $\varphi$ being a group homomorphism, it settles the first case. In all other cases, except for $m=2$ in the last formula, $G(t)$ is of the form $(1-F(t))$, with $F(t) \gg 2 t^{2}+t^{3}$, hence by [Gu, (4.2)(ii)],

$$
\varphi(G(t)) \gg \varphi\left(1-2 t^{2}-t^{3}\right) .
$$

In the last case $G(t)$ can be written $\left(1-t^{2}\right)\left(1-t^{2}-t^{2}\left(1+t^{2}+t^{3}\right)(1-t)^{-1}\right)$, which by the same result of Gulliksen implies

$$
\varphi(G(t)) \gg t^{2}+t^{2}\left(1+t^{2}+t^{3}\right)(1-t)^{-1}
$$

Finally we prove (iii) ${ }^{\circ}$ under condition (4.1). When $e_{2}(A)=3$, the inequality $r_{A} \leqslant \sqrt{2} / 2$ is seen by direct inspection of the power series (4.3). When $e_{2}(A)>4, L_{A}$ contains a free Lie subalgebra on at least 3 generators of degree 3 , hence $P_{A}(t) \gg$ $\left(1-3 t^{3}\right)^{-1}$, which implies $r_{A} \leqslant 3^{-1 / 3}<2^{-1 / 2}$. When $e_{2}(A)=4$ and $\operatorname{dim}_{k} H_{1}^{2}(K) \leqslant$ 4, Lemma 3.9 and Proposition 3.6(b) imply that $L_{A}$ contains a free Lie subalgebra on two generators of degree 2 , hence $r_{A} \leqslant \sqrt{2} / 2$ as above.

In the last case to remain undecided, $e_{2}(A)=4$ and no two linearly independent elements of $V=H_{1}(K)$ have zero product. Since under our condition (4.1) Lemma 
3.2 implies $V^{3}=0$, it is easy to see that for a suitably chosen basis $v_{1}, v_{2}, v_{3}, v_{4}$ of $V$, the subalgebra $W$ of $H(K)$, generated by $V$, is isomorphic to one of the following $k$-algebras:

$$
W^{\prime}=\Lambda(V) /\left(\Lambda^{3} V\right), \text { or } \quad W^{\prime \prime}=\Lambda(V) /\left(v_{1} v_{2}+v_{3} v_{4}\right) .
$$

I do not know shorter ways to finish the proof of the theorem than to compute the Poincaré series $P_{W}(t)=\Sigma_{n \geqslant 0} \Sigma_{p+q=n} \operatorname{dim}_{k} \operatorname{Tor}_{p, q}^{W}(k, k) t^{n}$ (here $p$ denotes the homological and $q$ the internal degrees of the components of the graded Tor functor).

Consider the divided powers algebra $U=U^{\prime}$ (resp. $U=U^{\prime \prime}$ ) obtained by adjoining to $U^{\prime}$ (resp. $U^{\prime \prime}$ ) variables $S_{1}, S_{2}, S_{3}, S_{4}$ of bidegree $(1,1)$ with differential mapping $S_{i}$ to $v_{i}$ (cf. [GL]). The bigraded homology of $U$ is computed by counting Euler characteristics in the complexes of vector spaces

$$
0 \leftarrow U_{q-2, q} \stackrel{d_{q-1, q}}{\leftarrow} U_{q-1, q} \stackrel{d_{q, q}}{\leftarrow} U_{q, q} \leftarrow 0 .
$$

In bidegrees $\neq(0,0)$, the result is

$$
\begin{aligned}
& \operatorname{dim}_{k} H_{p, q}\left(U^{\prime}\right)=\left\{\begin{array}{l}
0 \text { if } p \neq q-2, q \geqslant 1, \\
(q+1)(q-1)(q-2) / 2 \quad \text { if } p=q-2 \geqslant 1 ;
\end{array}\right. \\
& \operatorname{dim}_{k} H_{p, q}\left(U^{\prime \prime}\right)= \begin{cases}0 & \text { if } p \neq q-2, q \geqslant 1,(p, q) \neq(1,2), \\
1 & \text { if }(p, q)=(1,2), \\
(q+1)(q-1)(q-3) / 3 & \text { if } p=q-2 \geqslant 2 .\end{cases}
\end{aligned}
$$

For dimensional reasons, it is easy to see that any basis of $I H\left(U^{\prime}\right)$ (resp. of $I H\left(U^{\prime \prime}\right)$ ) admits a trivial Massey operation. Applying the (graded analogue of) Golod's theorem [Go 1], cf. also Corollary 1.6, we obtain after a computation with formal power series the following expressions:

$$
\begin{aligned}
& P_{W^{\prime}}(t)=\left(\left(1-t^{2}\right)^{4}+\left(t^{2}-4\right) t^{5}\right)^{-1}, \\
& P_{W^{\prime \prime}}(t)=\left(\left(1-t^{2}\right)^{4}\left(1-t^{3}-t^{4}\right)+\left(t^{4}-4 t^{2}+1\right) t^{3}\right)^{-1} .
\end{aligned}
$$

It remains to observe that, since $I^{3} W=0, W$ is an algebra retract of $H(K)$, hence $P_{W}(t) \ll P_{H(K)}(t)$. Together with the equality $P_{A}(t)=(1+t)^{3} P_{H(K)}(t)$ (e.g. [Av 1,(5.11)]), this gives $r_{A} \leqslant r_{W}<\sqrt{2} / 2$, and the proof of Theorem 1.2 is now complete.

5. Some relations between the conjectures.

(5.1) Proof of Proposition 0.6. Assume $\mathrm{C}_{5}$ holds. Then

$$
\chi_{2 i}(A) \leqslant \chi_{2 i}\left(A_{\mathfrak{p}}\right)+\operatorname{dim} A / \mathfrak{p} \leqslant \chi_{2 i}\left(A_{\mathfrak{p}}\right)+\operatorname{dim} A-\operatorname{dim} A_{\mathfrak{p}},
$$

which is the assertion of $\mathrm{C}_{4}(\mathrm{c})$. Furthermore, the identity

$$
e_{r}(A)-e_{r}\left(A_{\mathfrak{p}}\right)=(-1)^{r-1}\left(\chi_{r}(A)-\chi_{r}\left(A_{\mathfrak{p}}\right)\right)+(-1)^{r}\left(\chi_{r-1}(A)-\chi_{r-1}\left(A_{\mathfrak{p}}\right)\right)
$$

shows that $\mathrm{C}_{5}$ implies $\mathrm{C}_{6}$.

For $i>1, \mathrm{C}_{7}$ obviously follows from $\mathrm{C}_{8}$, while the inequality $e_{1}(A) \leqslant e_{1}(B)$ is known from $[\mathbf{L}]$. 
The fact that $\mathrm{C}_{6}$ is a consequence of $\mathrm{C}_{7}$ has been established in the proof of Theorem (2.6). ${ }^{10}$

Assume $\mathrm{C}_{4}(\mathrm{a})$ holds for artinian rings and $\mathrm{C}_{4}(\mathrm{c})$ holds for complete rings. Let $\mathfrak{p}$ be a prime ideal of $A$ and pick a prime ideal $\mathfrak{p}^{\prime}$ of $\hat{A}$ minimal over $\mathfrak{p}$. We have a local flat extension $A_{\mathfrak{p}} \rightarrow A_{\mathfrak{p}^{\prime}}$ with artinian fibre $\mathrm{C}=A_{\mathfrak{p}^{\prime}} / \mathfrak{p} A_{\mathfrak{p}^{\prime}}$. The following chain of inequalities establishes $\mathrm{C}_{4}(\mathrm{c})$ :

$$
\begin{aligned}
& \operatorname{dim} A_{\mathfrak{p}}-\chi_{2 i}\left(A_{\mathfrak{p}}\right) \\
& \leqslant \operatorname{dim} A_{\mathfrak{p}}-\chi_{2 i}\left(A_{\mathfrak{p}}\right)+\operatorname{dim} C-\chi_{2 i}(C) \quad\left(\text { by the } \operatorname{artinian} \mathrm{C}_{4}(\mathrm{a})\right) \\
& =\operatorname{dim} \hat{A}_{\mathfrak{p}^{\prime}}-\chi_{2 i}\left(\hat{A}_{\mathfrak{p}^{\prime}}\right) \quad \text { (by Theorem }(1.1)^{11} \text { ) } \\
& \leqslant \operatorname{dim} \hat{A}-\chi_{2 i}(\hat{A}) \\
& =\operatorname{dim} A-\chi_{2 i}(A) \text {. }
\end{aligned}
$$

Furthermore, taking a minimal prime $\mathfrak{p}$ of $A$, the already established $\mathrm{C}_{4}(\mathrm{c})$ and the artinian case of $\mathrm{C}_{4}(\mathrm{a})$ imply $\mathrm{C}_{4}(\mathrm{a})$ in general.

RemarK 5.2. Recall that Gulliksen [Gu, (3.3)] has proved $\mathrm{C}_{5}$ for homomorphic images of regular local rings and $r<\pi(A)$, hence $\mathrm{C}_{4}(\mathrm{c})$ is known for complete rings and all $i$, when char $k=0$, and for $i<$ char $k$ otherwise.

(5.3) Proof of Proposition 0.7. The implication $\mathrm{C}_{0} \Rightarrow \mathrm{C}_{1}$ is [ $\left.\mathbf{G u},(2.5)\right]$.

The assertion of $\mathrm{C}_{2}$ can be written in the form $\left(1-2 t^{q}\right)^{-1} \ll p \cdot P_{A}(t)$ for some positive integers $p$ and $q$. This implies the inequality $r_{A} \leqslant 2^{-1 / q}$, hence $C_{1}$.

Assuming $\mathrm{C}_{10}$, let $l_{1}$ and $l_{2}$ be free generators of $L, l_{1} \neq l_{2}$. If $\operatorname{deg} l_{i}$ is odd, we can replace $l_{i}$ by $\kappa\left(l_{i}\right)$, where $\kappa$ is the adjusting operator of $L$ (cf. [Sj]; note that if char $k \neq 2$, then $\left.\kappa\left(l_{i}\right)=1 / 2\left[l_{i}, l_{i}\right]\right)$. The graded Lie subalgebra of $L$ generated by (the new) $l_{1}, l_{2}$ is free as a graded subalgebra of a free Lie algebra, ${ }^{12}$ and since it is situated in even degrees, is a Lie algebra in the usual sense (i.e. $[a, b]=-[b, a]$ for any pair of elements). Then $\left[l_{1},\left[l_{2},\left[l_{2},\left[l_{2}, l_{1}\right]\right]\right]\right]$ and $\left[\left[l_{2}, l_{1}\right],\left[l_{2},\left[l_{2}, l_{1}\right]\right]\right]$ are distinct elements from its Hall basis, ${ }^{13}$ having equal degrees, say $q$. Applying Lemaire ${ }^{14}$ once more, we see they generate a free subalgebra. Passing to the universal envelopes, we have, for the Poincare series, $\left(1-2 t^{q}\right)^{-1} \ll P_{A}(t)$, hence $\mathrm{C}_{2}$ follows.

Finally, the implication $\mathrm{C}_{10} \Rightarrow \mathrm{C}_{9}$ is simply faithfully flat descent, by remarking that $E_{A}$ is free over the universal envelope $U(L)$ of $L$, which by assumption is the tensor algebra on a (graded) vector space of dimension $>1$, hence is not noetherian.

ACKNOWLEDGEMENTS. I want to thank E. S. Golod for motivating this research through joint work, in which the inequality $r_{A} \leqslant \sqrt{2} / 2$ was initially proved for rings with edim $A-\operatorname{depth} A \leqslant 3$, using a different approach.

\footnotetext{
${ }^{10}$ See footnote 2 .

${ }^{11}$ See footnote 2 .

12 J.-M. Lemaire, Algèbres connexes et homologie des éspaces de lacets, Lecture Notes in Math., no. 422 , Springer-Verlag, Berlin, 1974 (Theorem A.1.10).

${ }^{13}$ N. Bourbaki, Groupes et algèbres de Lie. Chap. II: Algèbres de Lie libres, Hermann, Paris, 1972.

${ }^{14}$ See footnote 12.
} 
A considerable part of this paper was worked out while the author was visiting the Institute Mittag-Leffler under the exchange programme between the Bulgarian Academy of Sciences and the Swedish Academy of Sciences. I want to thank Dan Laksov and the Institute for the stimulating atmosphere, and also to thank Jan-Erik Roos and Jorgen Backelin from the University of Stockholm for their interest in this work.

\section{REFERENCES}

[An] M. André, Hopf algebras with divided powers, J. Algebra 18 (1971), 19-50.

[Av 1] L. L. Avramov, On the Hopf algebra of a local ring, Izv. Akad. Nauk SSSR Ser. Mat. 38 (1974), 253-277 = Math. USSR-Izv. 8 (1974), 259-284.

[Av 2] __ Small homomorphisms of local rings, J. Algebra 50 (1978), 400-453.

[Av 3] _ Poincare series of almost complete intersections of embedding dimension three, PLISKA Stud. Math. Bulgar. 2 (1981), 167-172.

[Av 4] __ Sur la croissance des nombres de Betti d' un anneau local, C. R. Acad. Sci. Paris, Ser. A-B 289 (1979), A369-A372.

[AG] L. L. Avramov and E. S. Golod, On the homology of the Koszul complex of a local Gorenstein ring, Mat. Zametki 9 (1971), 53-58 = Math. Notes 9 (1971), 30-32.

[BK] W. Borho and H. Kraft, Über die Gelfand-Kirilloo dimension, Math. Ann. 220 (1976), 1-24.

[Ch] K.-T. Chen, Free subalgebras of loop space homology and Massey products, Topology 11 (1972), 237-243.

[Cl] A. Clark, Homotopy-commutativity and the Moore spectral sequence, Pacific J. Math. 15 (1965), 65-74.

[Go 1] E. S. Golod, On the homology of certain local rings, Dokl. Akad. Nauk SSSR 144 (1962), 479-482 = Soviet Math. Dokl. 3 (1962), 745-748.

[Go 2] __. On the homology of certain local rings, Uspehi Mat. Nauk 33 (1978), no. 5, 177-178.

[GM] V. K. A. M. Gugenheim and J. P. May, On the theory and applications of differential torsion products, Mem. Amer. Math. Soc. 142 (1974).

[Gu] T. H. Gulliksen, On the deviations of a local ring, Math. Scand. 47 (1980), 5-20.

[GL] T. H. Gulliksen and G. Levin, Homology of local rings, Queen's Papers in Pure and Appl. Math., No. 20, Queen's Univ., Kingston, Ont., 1969.

[L] C. Lech, Inequalities related to certain couples of local rings, Acta Math. 112 (1964), 69-89.

[M] J. P. May, Matric Massey products, J. Algebra 12 (1969), 533-568.

[Sc] G. Scheja, Über die Bettizahlen lokaler Ringe, Math. Ann. 155 (1964), 155-172.

[Sj] G. Sjödin, Hopf algebras and derivations, J. Algebra 64 (1980), 218-229.

[Sh] J. Shamash, The Poincare series of a local ring, J. Algebra 12 (1969), 453-470.

[W] H. Wiebe, Über homologische Invarianten lokaler Ringe, Math. Ann. 179 (1969), 257-274.

Institute of Mathematics, Bulgarian Academy of Sciences, P. O. Box 373, 1090 Sofia, BULGARIA 